\title{
ABSOLUTES OF ALMOST REALCOMPACTIFICATIONS
}

\author{
MOHAN L. TIKOO
}

(Received 16 April 1984; revised 28 September 1984)

Communicated by H. Rubinstein

\begin{abstract}
Given Hausdorff spaces $X$ and $Y$ and a perfect irreducible and $\theta$-continuous map $f$ from $X$ onto $Y$, a technique that carries open (ultra) filters on $X$ to open (ultra) filters on $Y$ back and forth in a natural way is introduced. It is proved that if $f$ is a perfect irreducible and $\theta$-continuous map from $X$ onto $Y$, then $X$ is almost realcompact if and only if $Y$ is almost realcompact. Several commutativity relations between the 'absolutes of almost realcompactifications' and the 'almost realcompactifications of absolutes' of a space $X$ are discussed.
\end{abstract}

1980 Mathematics subject classification (Amer. Math. Soc.): 54 D 25.

Keywords and phrases: absolutes, $H$-closed spaces, almost realcompact spaces.

\section{Preliminaries}

All spaces considered in this paper are Hausdorff. Throughout, $\tau(X)$ will denote the topology on the space $X$. If $X$ is a space and $A$ is a subset of $X$, then int ${ }_{X}(A), \mathrm{cl}_{X}(A)$ and bd ${ }_{X}(A)$ will denote the interior of $A$, closure of $A$, and the boundary of $A$ in $X$, respectively. A subset $A$ of a space $X$ is called regular open (respectively, regular closed) if $A=$ int $_{X} \mathrm{cl}_{X}(A)$ (respectively, $A=\mathrm{cl}_{X}$ int $_{X}(A)$.) The family $R O(X)$ (respectively, $R(X)$ ) denotes the complete Boolean algebra of regular open (respectively, regular closed) subsets of $X$, and $C O(X)$ denotes the algebra of all clopen (= closed and open) subsets of $X$. A space $X$ is called extremally disconnected if $\mathrm{cl}_{X}(U)$ is open in $X$ for each $U \in \tau(X)$. A space $X$ is called zero-dimensional if it has a basis consisting of clopen subsets. A map from a space $X$ to a space $Y$ is a (not necessarily continuous) function $f: X \rightarrow Y$. The

(C) 1986 Australian Mathematical Society $0263-6115 / 86 \$ A 2.00+0.00$ 
notation $f: X \rightarrow Y$ will mean that $f$ is a surjection. Let $f: X \rightarrow Y$. If $Z \subseteq X$, the small image of $A$ (under $f$ ) is defined (see [3], [14]) by $f^{\#}(A)=\{y \in Y$ : $\left.f^{\leftarrow}(Y) \subseteq A\right\}(\subseteq f(A))$. If $f$ is onto, then $f^{\#}(A)=Y \backslash f(X \backslash A)$. For a family $\mathscr{A} \subseteq \mathbf{P}(X), f^{\#}(\mathscr{A})$ will denote the family $\left\{f^{\#}(A): A \in \mathscr{A}\right\}$. A map $f: X \rightarrow Y$ is called compact if, for each $y \in Y, f^{\leftarrow}(y)$ is a compact subset of $X$, perfect if $f$ is both a compact and a closed mapping, and irreducible if $f$ is onto and, for each proper closed subset $A$ of $X, f(A) \neq Y$. A mapping $f: X \rightarrow Y$ is called $\theta$-continuous at a point $x \in X$ if, for each open neighborhood $G$ of $f(x)$ in $Y$, there is an open neighborhood $U$ of $x$ in $X$ such that $f\left(\operatorname{cl}_{X}(U)\right) \subseteq \operatorname{cl}_{Y}(G)$. If $f$ is $\theta$-continuous at each $x \in X$, then $f$ is called $\theta$-continuous (see [4]). A bijection $f$ : $X \rightarrow Y$ is called a $\theta$-homeomorphism provided that both the maps $f$ and $f^{-}$are $\theta$-continuous. Certainly, every continuous function is $\theta$-continuous. If $f: X \rightarrow Y$ is $\theta$-continuous and $Y$ is regular, then $f$ is continuous. Also, the composition of two $\theta$-continuous functions is $\theta$-continuous. Note that the next two facts contain well known results about maps.

(1.1) FACTS ( see [3], [14], [20]).

(a) Continuous maps with compact domain are perfect.

(b) If $f: X \rightarrow Y$ is a perfect map, and if $C$ is a compact subset of $Y$, then $f^{\leftarrow}(C)$ is a compact subset of $X$.

(c) The composition of two perfect (respectively, irreducible and closed) maps is a perfect (respectively, irreducible and closed) map.

(d) If $f: X \rightarrow Y$ is a perfect map, then

(i) for each closed subset $A$ of $X,\left.f\right|_{A}$ is a perfect mapping (whether regarded as a function into $Y$ or as a function onto $f(A)$ ), and

(ii) if $B \subseteq Y$, then $\left.f\right|_{f^{\leftarrow}(B)}: f^{\leftarrow}(B) \rightarrow B$ is a perfect mapping.

(e) If $f: X \rightarrow Y$ is a closed and irreducible surjection, and if $S$ is a dense subset of $Y$, then $f^{\leftarrow}(S)$ is a dense subset of $X$, and $\left.f\right|_{f^{-}(S)}$ is a closed and irreducible surjection from $f^{\leftarrow}(S)$ onto $S$.

(f) If $f: X \rightarrow Y$ is a perfect surjection, then there is a closed subset $C$ of $X$ such that $\left.f\right|_{C}: C \rightarrow Y$ is irreducible.

(g) A surjection $f: X \rightarrow Y$ is closed and irreducible if and only if $f^{\#}(G)$ is a non-empty open subset of $Y$ for each non-empty open subset $G$ of $X$.

(h) If $f: X \rightarrow Y$ is $\theta$-continuous, then for each $U \in \tau(Y), f^{-}\left(\operatorname{cl}_{Y}(U)\right)$ is a neighborhood of $f^{\leftarrow}(U)$, and $f\left[\mathrm{cl}_{X}\left(f^{\leftarrow}(U)\right)\right] \subseteq \mathrm{cl}_{Y}(U)$.

(1.2) FACTS (see [3], [14], [20]). Let $f: X \rightarrow Y$ be a closed, irreducible and $\theta$-continuous mapping.

(a) If $U \in \tau(Y)$, then $f\left[\mathrm{cl}_{X}\left(f^{\leftarrow}(U)\right)\right]=\operatorname{cl}_{Y}(U)$ and $\operatorname{int}_{X}\left[f^{\leftarrow}\left(\mathrm{cl}_{Y}(U)\right)\right]=$ int ${ }_{X} \mathrm{cl}_{X}\left(f^{\leftarrow}(U)\right)$. 
(b) If $G \in \tau(X)$, then $\operatorname{int}_{X} \mathrm{cl}_{X}(G)=\operatorname{int}_{X}\left[f^{\leftarrow}\left(\mathrm{cl}_{Y}\left(f^{\#}(G)\right)\right)\right] \subseteq \operatorname{cl}_{X}(G) \subseteq$ $f^{\leftarrow}\left[\mathrm{cl}_{Y}\left(f^{\#}(G)\right)\right]$.

(c) If $G \in \tau(X)$, then $f\left(\mathrm{cl}_{X}(G)\right)=\mathrm{cl}_{Y}\left(f^{\#}(G)\right)$.

(d) For each $U, V \in R O(Y)$, int ${ }_{X} \operatorname{cl}_{X}\left(f^{\leftarrow}(U \cap V)\right)=\operatorname{int}_{X} \mathrm{cl}_{X}\left(f^{\leftarrow}(U)\right) \cap$ int ${ }_{X} \mathrm{cl}_{X}(f \leftarrow(V))$.

(e) For each $W \in \tau(Y), f^{\#}\left[\operatorname{int}_{X} \mathrm{cl}_{X}\left[f^{\leftarrow}\left(\right.\right.\right.$ int $\left.\left.\left._{Y} \mathrm{cl}_{Y}(W)\right)\right)\right]=\operatorname{int}_{Y} \mathrm{cl}_{Y}(W)$.

(f) If $f$ is also perfect, then for each $G$ in $R O(X), f^{\#}(G) \in R O(Y)$.

(1.3) An open filter on a space $X$ will always mean a filter in the lattice $\tau(X)$ of all open subsets of $X$. A subcollection $\mathscr{B}$ of $\tau(X)$ is said to be an open filter base for some open filter on $X$ if for every $B_{1}, B_{2} \in \mathscr{B}$, there exists a $B \in \mathscr{B}$ such that $B \subseteq B_{1} \cap B_{2}$. If $\mathscr{A}$ is any non-empty family of open subsets of $X$ with the finite intersection property, then $\langle\mathscr{A}\rangle$ will denote the open filter on $X$ generated by $\mathscr{A}$. An open ultrafilter on $X$ is an open filter on $X$ which is a maximal element (with respect to set inclusion) in the family of all open filters on $X$. It is proved in [21, Example $12 \mathrm{G}$ ] that an open filter $\mathscr{U}$ on a space $X$ is an open ultrafilter if and only if for each nonempty open subset $U$ of $X$, either $U \in \mathscr{U}$ or $X \backslash \mathrm{cl}_{X}(U) \in \mathscr{U}$, if and only if for each $V \in \tau(X)$, if $V \cap U \neq \varnothing$ for every $U \in \mathscr{U}$, then $V \in \mathscr{U}$. This characterization of open ultrafilters will be used subsequently without reference. Note that every open ultrafilter $\mathscr{U}$ is prime (i.e. $A \cup B \in \mathscr{U}$ implies that $A \in \mathscr{U}$ or $B \in \mathscr{U}$ for each $A, B$ in $\tau(X)$ ), and for a set $U \in \tau(X), U \in \mathscr{U}$ if and only if int ${ }_{X} \mathrm{cl}_{X}(U) \in \mathscr{U}$. If $\mathscr{F}$ is a filterbase on $X$, then $\operatorname{ad}_{X}(\mathscr{F})=$ $\cap\left\{\operatorname{cl}_{X}(F): F \in \mathscr{F}\right\}$ denotes the adherence of $\mathscr{F}$ in $X . \mathscr{F}$ is called free if $\operatorname{ad}_{X}(\mathscr{F})=\varnothing$; otherwise, $\mathscr{F}$ is called fixed. For an open filter $\mathscr{F}$ on $X$, we shall denote by $\mathscr{F}_{s}$ the open filter on $X$ generated by the filterbase int $_{X} \mathrm{cl}_{X}(A)$ : $A \in \mathscr{F}\}$, i.e.,

$$
\mathscr{F}_{s}=\left\langle\left\{\operatorname{int}_{X} \mathrm{cl}_{X}(A): A \in \mathscr{F}\right\}\right\rangle .
$$

The following fact is needed in the sequel.

(1.4) FACT $[16,1.9]$. Let $\mathscr{F}$ be an open filter on a space $X$.

(a) $\cap\{\mathscr{U}: \mathscr{U}$ is an open ultrafilter on $X, \mathscr{U} \supset \mathscr{F}\}=\left\{T \in \tau(X)\right.$ : int ${ }_{X} \mathrm{cl}_{X}(T) \in$ $\mathscr{F}\}$, and ad ${ }_{X}\left\{T \in \tau(X)\right.$ : int $\left.{ }_{X} \mathrm{cl}_{X}(T) \in \mathscr{F}\right\}=\operatorname{ad}_{X}(\mathscr{F})$.

(b) $\mathscr{F}$ is contained in a unique open ultrafilter $\mathscr{U}$ if and only if $\mathscr{U}_{s} \subseteq \mathscr{F} \subseteq \mathscr{U}$.

(c) $\operatorname{ad}_{X}(\mathscr{F})=\operatorname{ad}_{X}\left(\mathscr{F}_{s}\right)$.

(d) If $\mathscr{U}$ is an open ultrafilter on $X$, then $\mathscr{U}_{s}$ is contained in a unique open ultrafilter on $X$, viz., $\mathscr{Q}$ itself.

We now prove two results which will be used frequently in the coming sections. (See for instance 1.15, 1.17, 1.21, 1.22, 2.2, 2.3, 2.13, 2.14 and 2.15.)

(1.5) Definition. Let $X$ and $Y$ be spaces and $f$ a map from $X$ onto $Y$. If $\mathscr{F}$ is an open filter on $X$ and $\mathscr{G}$ is an open filter on $Y$, we define

$$
\mathscr{F} \rightarrow=\left\{W \in \tau(Y): \operatorname{int}_{Y} \mathrm{cl}_{Y}(W) \supseteq f^{\#}(A) \text { for some } A \in \mathscr{F}\right\},
$$


and

$$
\mathscr{G}^{\leftarrow}=\left\{U \in \tau(X): f^{\#}(u) \in \mathscr{G}\right\} .
$$

(1.6) Proposition. Let $X$ and $Y$ be Hausdorff spaces and $f: X \rightarrow Y$ a perfect, irreducible and $\theta$-continuous surjection. If $\mathscr{F}$ is any open filter on $X$, then the following statements are true.

(a) $f^{\#}(\mathscr{F})$ is an open filter base on $Y$, and $f\left(\operatorname{ad}_{X}(\mathscr{F})\right)=\operatorname{ad}_{Y}\left(f^{\#}(\mathscr{F})\right)=$ $\operatorname{ad}_{Y}\left\langle f^{\#}(\mathscr{F})\right\rangle$.

(b) (i) $\mathscr{F} \rightarrow$ is an open filter on $Y$ containing $f^{\#}(\mathscr{F}), \mathscr{F} \rightarrow=\bigcap\{\mathscr{W}: \mathscr{W}$ is an open ultrafilter on $Y$ such that $\left.\mathscr{W} \supseteq f^{\#}(\mathscr{F})\right\}$, and $\operatorname{ad}_{Y}(\mathscr{F} \rightarrow)=\operatorname{ad}_{Y}\left(f^{\#}(\mathscr{F})\right)$.

(ii) Furthermore, if $\mathscr{F}$ is an open ultrafilter on $X$, then $\mathscr{F} \rightarrow$ is an open ultrafilter on $Y$.

Proof. Obviously, $f^{\#}(\mathscr{F})$ is an open filter base on $Y$. Also, $f\left(\operatorname{ad}_{X}(\mathscr{F})\right)=$ $f\left[\cap\left\{\operatorname{cl}_{X}(A): A \in \mathscr{F}\right\}\right] \subseteq \bigcap\left\{f\left(\operatorname{cl}_{X}(A)\right): A \in \mathscr{F}\right\}=\bigcap\left\{\operatorname{cl}_{Y}\left(f^{\#}(A): A \in \mathscr{F}\right)\right\}$ (by $1.2)=\operatorname{ad}_{Y}\left(f^{\#}(\mathscr{F})\right)$. To prove the reverse inequality, let $p \in \operatorname{ad}_{Y}\left(f^{\#}(\mathscr{F})\right)$. It suffices to show that $f^{\leftarrow}(p) \cap \operatorname{ad}_{X}(\mathscr{F}) \neq \varnothing$. Assume that $f^{\leftarrow}(p) \cap \operatorname{ad}_{X}(\mathscr{F})$ $=\varnothing$. Then $f^{\leftarrow}(p) \subseteq\left\{X \backslash \operatorname{cl}_{X}(A): A \in \mathscr{F}\right\}$ and, since $f^{-}(p)$ is a compact subset of $X$, there exist finitely many $A_{i} \in \mathscr{F}, i=1,2, \ldots, n$ such that $f^{\leftarrow}(p) \subseteq$ $\cup\left\{X \backslash \operatorname{cl}_{X}\left(A_{i}\right): i=1,2, \ldots, n\right\} \subseteq X \backslash \mathrm{cl}_{X}\left(\cap\left\{A_{i}: i=1,2, \ldots, n\right\}\right)=X \backslash \mathrm{cl}_{X}(A)$, where $A=\cap\left\{A_{i}: i=1,2, \ldots, n\right\} \in \mathscr{F}$. Thus $f^{\leftarrow}(p) \cap \mathrm{cl}_{X}(A)=\varnothing$, whence $p \notin f\left(\mathrm{cl}_{X}(A)\right)=\operatorname{cl}_{Y}\left(f^{\#}(A)\right)$, contradicting the fact that $p \in \operatorname{ad}_{Y}\left(f^{\#}(\mathscr{F})\right)$. Therefore, $p \in f\left(\operatorname{ad}_{X}(\mathscr{F})\right)$, and (a) follows. An easy verification shows that $\mathscr{F} \rightarrow$ is an open filter on $Y$ containing $f^{\#}(\mathscr{F})$, and the first equality in (b)(i) is a direct consequence of 1.4. The last part of (b)(i) follows from 1.4. To prove (b)(ii), let $V$ be an open subset of $Y$ such that $V \cap W \neq \varnothing$ for all $W \in \mathscr{F} \rightarrow$. Then $V \cap$ $f^{\#}(A) \neq \varnothing$ for each $A \in \mathscr{F}$. So, $\left(\operatorname{int}_{Y} \operatorname{cl}_{Y}(V)\right) \cap f^{\#}(A) \neq \varnothing$, whence int ${ }_{X} \mathrm{cl}_{X}\left(f^{\leftarrow}\left(\right.\right.$ int $\left.\left._{Y} \mathrm{cl}_{Y}(V)\right)\right) \cap A \neq \varnothing$ for each $A \in \mathscr{F}$. Using 1.2(e) and the fact that $\mathscr{F}$ is an open ultrafilter, we obtain $\operatorname{int}_{Y} \mathrm{cl}_{Y}(V) \in f^{\#}(\mathscr{F}) \subseteq \mathscr{F} \rightarrow$, whence $V \in \mathscr{F} \rightarrow$, and $\mathscr{F} \rightarrow$ is an open ultrafilter on $Y$. The proof of the proposition is complete.

(1.7) Proposition. Let $X$ and $Y$ be Hausdorff spaces and $f: X \rightarrow Y$ a perfect, irreducible and $\theta$-continuous surjection. If $\mathscr{G}$ is an open filter on $Y$, then the following statements are true.

(a) $\mathscr{G}^{\leftarrow}$ is an open filter on $X$ such that for every $U \in \mathscr{G}$, int ${ }_{X} \mathrm{cl}_{X}\left(f^{\leftarrow}(U)\right) \in$ $\mathscr{G}^{\leftarrow}$, and $f\left[\operatorname{ad}_{X}\left(\mathscr{G}^{\leftarrow}\right)\right]=\operatorname{ad}_{Y}(\mathscr{G})$.

(b) If $\mathscr{G}$ is an open ultrafilter on $Y$, then $\mathscr{G}^{\leftarrow}$ is an open ultrafilter on $X$.

Proof. It is easy to see that $\mathscr{G}^{\leftarrow}$ is an open filter on $X$. Now, if $U \in \mathscr{G}$, then by 1.2 and the fact that $U \subseteq f^{\#}\left(\operatorname{int}_{X}\left(f^{\leftarrow}\left(\operatorname{cl}_{Y}(U)\right)\right)\right.$, it follows that 
$f^{\#}\left(\right.$ int $\left._{X} \mathrm{cl}_{X}\left(f^{\leftarrow}(U)\right)\right) \in \mathscr{G}$. Hence int ${ }_{X} \mathrm{cl}_{X}\left(f^{-}(U)\right) \in \mathscr{G}^{\leftarrow}$. Now, let $p \in$ $f\left(\operatorname{ad}_{X}\left(\mathscr{G}^{-}\right)\right)$. If $p \notin \operatorname{ad}_{Y}(\mathscr{G})$, then there exists a regular open set $U \in \mathscr{G}$ and a regular open neighborhood $V$ of $p$ in $Y$ such that $V \cap U=\varnothing$. By 1.2, $G=$ int ${ }_{X} \mathrm{cl}_{X} f^{-}(V)$ is an open neighborhood of $f^{-}(p)$ in $X$ such that $G \cap$ int ${ }_{X} \mathrm{cl}_{X}\left(f^{-}(U)\right)=\varnothing$. Since int $\left.{ }_{X} \mathrm{cl}_{X} f^{-}(U)\right) \in \mathscr{G}^{-}$, it follows that $f^{-}(p) \cap$ $\operatorname{ad}_{X}\left(\mathscr{G}^{-}\right)=\varnothing$, contradicting the fact that $p \in f\left(\operatorname{ad}_{X}\left(\mathscr{G}^{-}\right)\right)$. Thus, $f\left(\operatorname{ad}_{X}\left(\mathscr{G}^{-}\right)\right)$ $\subseteq \operatorname{ad}_{Y}(\mathscr{G})$. To prove the reverse inequality, let $p \in \operatorname{ad}_{Y}(\mathscr{G})$. It suffices to show that $f^{-}(p) \cap \operatorname{ad}_{X}\left(\mathscr{G}^{-}\right) \neq \varnothing$. Assume that $f^{-}(p) \cap \operatorname{ad}_{X}\left(\mathscr{G}^{\leftarrow}\right)=\varnothing$. Then $f^{-}(p) \subseteq \bigcup\left\{X \backslash \mathrm{cl}_{X}(A): A \in \mathscr{G}^{-}\right\}$. Since $f^{-}(p)$ is compact, there exist finitely many $A_{i}, \quad i=1,2, \ldots, n$ in $\mathscr{G}^{-}$such that $f^{-}(p) \subseteq \bigcup\left\{X \backslash \mathrm{cl}_{X}\left(A_{i}\right): i=\right.$ $1,2, \ldots, n\}=B$ (say). Then, $A=\bigcap\left\{A_{i}: i=1,2, \ldots, n\right\} \in \mathscr{G}^{\leftarrow}$. Consequently, $f^{\#}(A) \in \mathscr{G}, f^{\#}(B)$ is an open neighborhood of $p$ in $Y$ and $f^{\#}(A) \cap f^{\#}(B)=\varnothing$, contradicting the fact that $p \in \operatorname{ad}_{Y}(\mathscr{G})$. Thus, $f^{-}(p) \cap \operatorname{ad}_{X}\left(\mathscr{G}^{-}\right) \neq \varnothing$, whence $p \in f\left(\operatorname{ad}_{X}\left(\mathscr{G}^{\leftarrow}\right)\right)$. Therefore, $\operatorname{ad}_{Y}(\mathscr{G}) \subseteq f\left(\operatorname{ad}_{X}\left(\mathscr{G}^{\leftarrow}\right)\right)$, and (a) follows. To prove (b) let $\mathscr{G}$ be an open ultrafilter on $Y$. If $B \in \tau(X)$ and $B \notin \mathscr{G}^{\leftarrow}$, then $f^{\#}(B) \notin \mathscr{G}$. Since $\mathscr{G}$ is an open ultrafilter, it follows that $f^{*}\left(X \backslash \operatorname{cl}_{X}(B)\right)=Y \backslash f\left(\mathrm{cl}_{X}(B)\right)=$ $Y \backslash \operatorname{cl}_{Y}\left(f^{\#}(B)\right) \in \mathscr{G}$. So $X \backslash \operatorname{cl}_{X}(B) \in \mathscr{G}^{\leftarrow}$. Hence, $\mathscr{G}^{\leftarrow}$ is an open ultrafilter on $X$.

(1.8) RemarK. A straightforward verification shows that if $X \rightarrow Y$ is a perfect, irreducible and $\theta$-continuous surjection, if $\mathscr{F}$ is an open ultrafilter on $X$, and if $\mathscr{G}$ is an open ultrafilter on $Y$, then

(a) $\mathscr{F}=(\mathscr{F} \rightarrow)^{\leftarrow}$, and

(b) $\mathscr{G}=\left(\mathscr{G}^{+}\right)^{\rightarrow}$.

(1.9) EXTENSIONS OF SPACES. An extension of a space $X$ is a pair $[Y, j]$, where $Y$ is a Hausdorff space and $j$ is a topological dense embedding of $X$ into $Y$. However, as is customary, we shall identify $j(x)$ with $x$ for each $x \in X$ and regard $X$ as a dense subspace of $Y$. Two extensions $Y_{1}$ and $Y_{2}$ of a space $X$ are called equivalent if there is a homeomorphism from one onto the other whose restriction to $X$ is the identity map $\iota_{X}$ on $X$. We identify two equivalent extensions of a space $X$. With this convention, if $\mathbf{E}(X)$ is the class of all the extensions of a space $X$, then $\mathbf{E}(X)$ is a set and $|\mathbf{E}(X)| \leqslant|\mathbf{P}(\mathbf{P}(\mathbf{P}(\mathbf{P}(X))))|$. If $Y$, $Z \in \mathbf{E}(X)$, then $Y$ is said to be projectively larger than $Z$ (written hereafter $Y \geqslant Z$ ) if there is a continuous mapping $\phi: Y \rightarrow Z$ such that $\left.\phi\right|_{X}=\iota_{X}$. If $\mathbb{P}$ is a topological property and $\mathbf{P}(X)=\{Y \in \mathbf{E}(X): Y$ has the property $\mathbb{P}\}$, then an element $Y$ of $\mathbf{P}(X)$ is called a projective maximum for $\mathbb{P}(X)$ if $Y \geqslant Z$ for each $Z \in \mathbf{P}(X)$. A projective maximum in $\mathbf{P}(X)$, if it exists, is unique.

(1.10) (See [12].) Let $Y$ be an extension of a space $X$.

(a) If $\mathscr{U}$ is an open (ultra) filter on $X$, then $\mathscr{U}^{*}=\{U \in \tau(Y): U \cap X \in \mathscr{U}\}$ is an open (ultra) filter on $Y$ which converges in $Y$ if and only if $\mathscr{U}$ converges in $Y$. 
(b) If $\mathscr{W}$ is an open (ultra) filter on $Y$, then $\mathscr{W}_{*}=\{W \cap X: W \in \mathscr{W}\}$ is an open (ultra) filter on $X$ which converges in $Y$ if and only if $\mathscr{W}$ converges in $Y$.

If more than one extension is involved, the meanings of $\mathscr{U}^{*}$ and $\mathscr{W}_{*}$ will be clear from the context.

(1.11) Let $Y \in \mathbf{E}(X)$. For a point $y \in Y$, let

$$
\mathcal{O}_{Y}^{y}=\left(\mathcal{N}_{y}\right)_{*}=\left\{U \cap X: U \in \mathscr{N}_{y}\right\}
$$

where $\mathscr{N}_{y}$ is the open neighborhood filter of $y$ in $Y$. For an open subset $U$ of $X$, let

$$
{ }^{o_{Y}}(U)=\left\{y \in Y: U \in \mathcal{O}_{Y}^{Y}\right\} \text {. }
$$

The family $\left\{o_{Y}(U): U \in \tau(X)\right\}$ forms an open base for a coarser Hausdorff topology $\tau^{\#}$ on $Y$. The space $\left(Y, \tau^{\#}\right)$, denoted by $Y^{\#}$ is an extension of $X$, and $\left(Y^{\#}\right)^{\#}=Y^{\#}$ (see [1], [15]).

Now, let $Y^{+}$be the space with the underlying set of $Y$ and the topology generated by the family $\mathscr{B}=\left\{U \cup\{y\}: y \in Y, U \in \mathcal{O O}_{Y}^{Y}\right\} \cup \tau(X)$. The space $Y^{+}$ is a Hausdorff extension of $X, \tau(Y) \subseteq \tau\left(Y^{+}\right), X$ is an open-dense subspace of $Y^{+}$, and $Y^{+} \backslash X$ is closed and discrete. Moreover, $\left(Y^{+}\right)^{+}=Y^{+}$. It is well known and easy to prove that for each $U \in \tau(X), \operatorname{cl}_{Y}(U)=\operatorname{cl}_{Y^{+}}(U)=\operatorname{cl}_{Y^{*}}(U)$ [15]. The following facts are used in the sequel.

(1.12) Facts (see [15]). Let $Y$ be an extension of a space $X$.

(a) For each $U \in \tau(X), U \subseteq o_{Y}(U)=Y \backslash \operatorname{cl}_{Y}(X \backslash U)=U\{W \in \tau(Y)$ : $W \cap$ $X \subseteq U\}$.

(b) If $W \in \tau(Y)$, then

(i) $X \cap o_{Y}(W \cap X)=W \cap X$,

(ii) $W \subseteq o_{Y}(W \cap X) \subseteq \operatorname{cl}_{Y}(W \cap X)=\operatorname{cl}_{Y}(W)=\operatorname{cl}_{Y}\left[o_{Y}(W \cap X)\right]=$ $\operatorname{cl}_{Y^{*}}\left[o_{Y}(W \cap X)\right]$,

(iii) int ${ }_{Y} \mathrm{cl}_{Y}(W)=$ int $_{Y} \mathrm{cl}_{Y}(W \cap X)=o_{Y}\left(\right.$ int $\left._{X} \mathrm{cl}_{X}(W \cap X)\right)$.

(c) For each $y \in Y,\left\{\mathcal{O}_{Y^{*}}^{*}: y \in Y^{\#}\right\}=\left\{\mathcal{O}_{Y}^{Y}: y \in Y\right\}=\left\{\mathcal{O}_{Y}^{Y}: y \in Y^{+}\right\}$.

(d) If $Z$ is an extension of $X$ such that $Z$ and $Y$ have the same underlying set, then $Y^{\#} \leqslant Z \leqslant Y^{+}$if and only if $\left\{\mathcal{O}_{Y}^{Y}: y \in Y\right\}=\left\{\mathcal{O}_{Z}: y \in Z\right\}$ and, in both cases, $Z^{\#}=Y^{\#}$, and $Z^{+}=Y^{+}$.

(e) For any space $Y \in \mathbf{E}(X),\left(Y^{\#}\right)^{+}=Y^{+}$and $\left(Y^{+}\right)^{\#}=Y^{\#}$.

An extension $Y$ of a space $X$ is called a simple (respectively strict) extension if $Y=Y^{+}$(respectively $Y=Y^{*}$ ). The extensions $Y^{+}$and $Y^{\#}$ were introduced by Banaschewski [1].

REMARK. If $X$ is a space, then $R O(X)$ forms an open base for a Hausdorff topology $\tau_{s}$ on $X$. The space $\left(X, \tau_{s}\right)$ is denoted by $X_{s}$ and is called the semiregularization of $X$ (see [15]). A space $X$ is semiregular if and only if $X=X_{s}$. If $Y \in \mathbf{E}(X)$, then $Y_{s} \in \mathbf{E}\left(X_{s}\right)$. Moreover if $Y \in \mathbf{E}(X)$ and $Y$ is semiregular, then $Y=Y^{\#}$. 
(1.13) The KatÉtov and Fomin EXTENSIONS. Recall that a space $X$ is $H$-closed provided that $X$ is closed in every Hausdorff space $Y$ in which $X$ is embedded. Let $X$ be a space and let $\mathbf{F}(X)=\{\mathscr{U}: \mathscr{U}$ is a free open ultrafilter on $X\}$. Let $\tilde{X}=X \cup \mathbf{F}(X)$. Define a topology $\tau^{+}$on $\tilde{X}$ as follows: $X$ is open in $\tilde{X}$, and for $\mathscr{U} \in \tilde{X} \backslash X$ a basic open neighborhood of $\mathscr{U}$ is $U \cup\{\mathscr{U}\}$ where $U$ is open in $X$ and $U \in \mathscr{U}$. Then $\left(\tilde{X}, \tau^{+}\right)$is a simple $H$-closed extension of $X$, denoted by $\kappa X$, and is called the Katětov extension of $X$ (see [9]). Now let $\tau^{\#}$ be another topology on $\tilde{X}$ generated by the (open basis) $\{o(U): U \in \tau(X)\}$, where $o(U)=U \cup\{\mathscr{U}$ $\in \tilde{X} \backslash X: U \in \mathscr{U}\}$. Then $\left(\tilde{X}, \tau^{\#}\right)$ is a strict $H$-closed extension of $X$, denoted by $\sigma X$, and is called the Fomin extension of $X$ (see [4]). For more details see [9], [15], [16].

(1.14) The absolute $E X$ of a SPACE $X$. A complete description and historical development of the theory of absolutes occurs in the survey paper of Woods [23]. Here we shall briefly summarize those properties of the absolutes which will be used subsequently in this paper. In what follows $\beta X$ (resp. $v X$ ) will denote the Stone-Čech compactifications (resp. Hewitt realcompactifications) of a Tychonoff space $X$.

Let $X$ be a Hausdorff space and let $E X$ be the set of all the convergent open ultrafilters on $X$. For each $U \in \tau(X)$, let

(a) $O_{X} U=\{\mathscr{F}: \mathscr{F} \in E X, U \in \mathscr{F}\}$.

The family $\left\{O_{X} U: U \in \tau(X)\right\}$ forms an open base for a Hausdorff topology $\tau(E X)$ on $E X$. The space $(E X, \tau(E X))$, denoted by $E X$ henceforth, is extremally disconnected and zero-dimensional, and it has the following properties (see [8], [13], [14], [19], [20] and [23]).

(b) For each $U \in \tau(X)$ and $\left\{U_{i}: \quad i \in \Lambda\right\} \subseteq \tau(X), \quad O_{X}\left(X \backslash \mathrm{cl}_{X}(U)\right)=$ $E X \backslash O_{X}(U)$, and $\mathrm{cl}_{E X}\left[\bigcup\left\{O_{X} U_{i}: i \in \Lambda\right\}\right]=O_{X}\left[\bigcup_{i \in \Lambda} U_{i}\right]$.

(c) The map $k_{X}: E X \rightarrow X$ given by $k_{X}(\mathscr{F})=a d_{X}(\mathscr{F})$ is well defined and is a perfect irreducible and $\theta$-continuous surjection such that $k_{X}\left(O_{X} U\right)=\mathrm{cl}_{X}(U)$ for all $U \in \tau(X)$.

(d) The space $E X$ is unique in the sense that if $Z$ is any extremally disconnected and zero-dimensional space, and $\phi: Z \rightarrow X$ is a perfect, irreducible and $\theta$-continuous surjection, then there is a homeomorphism $f: E X \rightarrow Z$ such that $\phi \circ f=k_{X}$. In this case we write $[Z, \phi] \equiv{ }_{X}\left[E X, k_{X}\right]$.

(e) The space $X$ is $H$-closed if and only if $E X$ is compact.

(f) $\beta E X=\mathrm{S}(R(X))$, the Stone space of the complete Boolean algebra $R(X)$. Moreover if $h X$ is any $H$-closed extension of $X$, then there exists a perfect, irreducible and $\theta$-continuous mapping from $\beta E X$ onto $h X$.

(g) $\beta E X \backslash E X \simeq \sigma X \backslash X$. In particular, $\sigma X \backslash X$ is zero-dimensional.

(h) (i) If $U \in \tau(X)$, then $k_{X}^{\#}\left(O_{X} U\right)=\operatorname{int}_{X} \mathrm{cl}_{X} U$. 
(ii) If $W \in \tau(E X)$, then $\mathrm{cl}_{X}\left(k_{X}(W)\right)=\mathrm{cl}_{X}\left(k_{X}^{\#}(W)\right)$, and there is a set $V \in R O(X)$ such that $\mathrm{cl}_{E X}(W)=O_{X} V$. Furthermore, $\operatorname{cl}_{X}\left(k_{X}^{\#}(W)\right)=\mathrm{cl}_{X}(V)$ and $O_{X}\left(k_{X}^{\#}(W)\right)=O_{X} V$.

(i) Let $U$ be an open subset of the space $X$. Let $k_{U}: O_{X} U \rightarrow \mathrm{cl}_{X} U$ be defined by $k_{U}=\left.k_{X}\right|_{o_{X} U}$. Then $k_{U}$ is a perfect, irreducible and $\theta$-continuous mapping from $O_{X} U$ onto $\mathrm{cl}_{X}(U)$.

An immediate application of 1.6, 1.7, and 1.8 leads to a short proof of a well known theorem proved by Iliadis and Fomin [8].

(1.15) THEOREM. Let $X$ and $Y$ be Hausdorff spaces such that there exists a perfect, irreducible and $\theta$-continuous mapping $f: X \rightarrow Y$. Then there exists a homeomorphism $\phi: E X \rightarrow E Y$ such that $k_{Y} \circ \phi=f \circ k_{X}$.

Proof. Let $\mathscr{U}, \mathscr{V}$, be two distinct members of $E X$. Then there exist open sets $U \in \mathscr{U}$ and $V \in \mathscr{V}$ such that $U \cap V=\varnothing$. Now $f^{\#}(U) \in \mathscr{U}^{\rightarrow}$ (see 1.6), $f^{\#}(V)$ $\in \mathscr{V}^{\rightarrow}$ and $f^{\#}(U) \cap f^{\#}(V)=\varnothing$. Hence, $\mathscr{U} \rightarrow \neq \mathscr{V} \rightarrow$. Moreover, by 1.6, $\mathscr{U}^{\rightarrow}$ $\in E Y$ for each $\mathscr{U} \in E X$. Thus, $\phi: E X \rightarrow E Y$, defined by $\phi(\mathscr{U})=\mathscr{U} \rightarrow$, is a one-to-one mapping from $E X$ into $E Y$. By 1.8, $\phi$ is onto, and $k_{Y} \circ \phi=f \circ k_{X}$. Let $O_{X} U$ be a basic open set in $E X$. Then $\mathscr{U} \in O_{X} U$ if and only if $U \in \mathscr{U}$, if and only if int ${ }_{X} \mathrm{cl}_{X}(U) \in \mathscr{U}=\left(\mathscr{U}^{\leftarrow}\right)^{\rightarrow}$, if and only if $f^{\#}\left(\right.$ int $\left._{X} \mathrm{cl}_{X}(U)\right) \in \mathscr{U}^{\leftarrow}$, if and only if $\phi(\mathscr{U})=\mathscr{Q} \rightarrow \in O_{Y}\left(f^{\#}\left(\operatorname{int}_{X} \mathrm{cl}_{X}(U)\right)\right)$, which is open in $E Y$. Thus, $\phi\left(O_{X} U\right)=O_{Y}\left(f^{\#}\left(\right.\right.$ int $\left._{X} \mathrm{cl}_{X}(U)\right)$, and since $\phi$ is a bijection it follows that $\phi$ is an open mapping. To show that $\phi$ is continuous, let $O_{Y} V$ be a basic open set in $E Y$. Note that $O_{Y} V=O_{Y}\left(\right.$ int $\left._{X} \mathrm{cl}_{X}(V)\right)=O_{Y}\left[f^{\#}\left(\operatorname{int}_{X} \mathrm{cl}_{X}\left(f^{\leftarrow}\left(\right.\right.\right.\right.$ int $\left.\left.\left.\left._{Y} \mathrm{cl}_{Y}(V)\right)\right)\right)\right]=$ $\phi\left[O_{X}\left[\operatorname{int}_{X} \operatorname{cl}_{X}\left(f^{\leftarrow}\left(\operatorname{int}_{Y} \mathrm{cl}_{Y}(V)\right)\right)\right]\right]$. Since $\phi$ is a bijection, the set $\phi^{\leftarrow}\left(O_{Y}(V)\right)=$ $O_{X}\left[\right.$ int $\left._{X} \mathrm{cl}_{X}\left(f^{\leftarrow}\left(\operatorname{int}_{Y} \mathrm{cl}_{Y}(V)\right)\right)\right]$ is open. Hence $\phi$ is continuous, and the proof of the theorem is complete.

Two spaces $X$ and $Y$ are called co-absolute if $E X \simeq E Y$.

(1.16) The ABsolute $P X$. Banaschewski [2] and Mioduschewski and Rudolf [13] constructed another absolute $P X$ where the underlying set is $E X$ but the topology $\tau(P X)$ is generated by the family $\left\{O_{X} U \cap k_{X}(V): U, V \in \tau(X)\right\}$. It turns out that the space $(P X, \tau(P X))$, written as $P X$ henceforth, is extremally disconnected and Hausdorff, but is no longer Tychonoff in general. However, the map $k_{X}$ (which we now denote by $\pi_{X}: P X \rightarrow X$ ) is continuous as well as perfect and irreducible. Clearly $X$ is $H$-closed if and only if $P X$ is $H$-closed, and $\left[P X, \pi_{X}\right] \equiv{ }_{X}\left[E X, k_{X}\right]$ if and only if $X$ is regular. Also, $E X=(P X)_{s}$, and $R O(E X)=R O(P X)=C O(E X)=\left\{O_{X} U: U \in \tau(X)\right\}$ (see [19]). Moreover, $\left[P X, \pi_{X}\right]$ is unique in the sense that if $Z$ is any extremally disconnected space and $\mathrm{g:} Z \rightarrow X$ is a perfect, irreducible, continuous surjection, then there is homeomorphism $h: P X \rightarrow Z$ such that $g \circ h=\pi_{X}$. 
The following results will be used later.

(1.17). Lemma. Let $\alpha$ be an open ultrafilter on EX (respectively, PX). Then $\alpha \rightarrow=\left\{W \in \tau(X): O_{X} W \in \alpha\right\}$.

Proof. Let $\mathscr{W}=\left\{W \in \tau(X): O_{X} W \in \alpha\right\}$. To show that $\alpha \vec{a}=\mathscr{W}$, let $W \in \mathscr{W}$. Then $O_{X} W \in \alpha$. Since int ${ }_{X} \mathrm{cl}_{X}(W)=k_{X}^{*}\left(O_{X} W\right) \in k_{X}^{\#}(\alpha) \subseteq \alpha \overrightarrow{ }$ (see 1.6 and

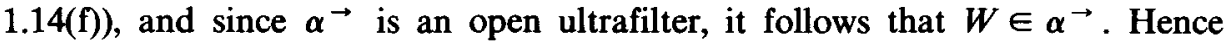
$\mathscr{W} \subseteq \alpha^{\rightarrow}$. To prove the reverse inequality, let $W \in \alpha^{\rightarrow}$. Then there is a set $A \in \alpha$ such that $k_{X}^{\#}(A) \subseteq$ int $_{X} \mathrm{cl}_{X}(W)$. Now $O_{X}\left(k_{X}^{\#}(A)\right) \subseteq O_{X}\left(\right.$ int $\left._{X} \mathrm{cl}_{X}(W)\right)=O_{X} W$, and $O_{X}\left(k_{X}^{\#}(A)\right)=\mathrm{cl}_{E X}(A)$ (see 1.14(f)). Since $E X$ is extremally disconnected, $\operatorname{cl}_{E X}(A) \in \alpha$. Hence, $O_{X} W \in \alpha$, and therefore $W \in \mathscr{W}$. Thus $\alpha \rightarrow \mathscr{W}$. The same argument holds for $P X$, and the result follows.

(1.18) Lemma. Let $X$ be a space and $\mathscr{U} \in E X$ (respectively $\mathscr{U} \in P X$ ). If $G$ is open in $E X$ (respectively $P X$ ), and if $\mathscr{U} \in G$, then $k_{X}^{\#}(G) \in \mathscr{U}$ (respectively $\left.\pi_{X}(G) \in \mathscr{U}\right)$.

Proof. Suppose $k_{X}^{\#}(G) \notin \mathscr{U}$. Since $k_{X}^{\#}(G) \in \tau(X)$, and since $\mathscr{U}$ is an open ultrafilter on $X$, there exists a set $A \in \mathscr{U}$ such that $k_{X}^{\#}(G) \cap A=\varnothing$. Therefore, by $1.14(\mathrm{f}), \mathrm{cl}_{E X} G \cap O_{X} A=\varnothing$. Hence $G \cap O_{X} A=\varnothing$, which is impossible since $\alpha \in G$ and $\alpha \in O_{X} A$. This proves the result for $E X$. For $P X$, the result follows by the same proof using 1.16 and the fact that for any space $Z$, and any open subset $U$ of $Z, \mathrm{cl}_{Z}(U)=\mathrm{cl}_{Z_{s}}(U)$.

(1.19) LEMMA. Let $X$ be a space such that every closed and nowhere dense subset of $E X$ is compact. Let $G$ be a nonempty open subset of $E X$ and $\alpha \in o_{\sigma E X}(G)$. Then there exists an open subset $U$ of $X$ such that $\alpha \in o_{\sigma E X}\left(O_{X} U\right) \subseteq 0_{\sigma E X}(G)$.

Proof. If $\alpha \in o_{\sigma E X}\left(O_{X} G\right) \cap E X=G$, there is an open subset $U$ of $X$ such that $\alpha \in O_{X} U \subseteq G$. Hence $\alpha \in o_{\sigma E X}\left(O_{X} U\right) \subseteq o_{\sigma E X}(G)$. If $\alpha \in o_{\sigma E X}(G) \backslash E X$, then $G \in \alpha$. By 1.14(f), there is a regular open subset $U_{0}$ of $X$ such that $\operatorname{cl}_{E X}(G)=$ $O_{X} U_{0}$. Also, $\mathrm{cl}_{E X}(G) \backslash G$ is compact by hypothesis. Let $p \in \mathrm{cl}_{E X}(G) \backslash G$. Since $\alpha$ is a free open ultrafilter on $E X$, there is a set $A_{p} \in \alpha$ such that $p \notin \operatorname{cl}_{E X}\left(A_{p}\right)$ $=O_{X} U_{p}$ for some $U_{p} \in R O(X)$. So $p \in E X \backslash O_{X} U_{p}$. Hence $\left\{E X \backslash O_{X} U_{p}: p \in\right.$ $\left.\operatorname{cl}_{E X}(G) \backslash G\right\}$ is an open covering of the compact set $\mathrm{cl}_{E X}(G) \backslash E X$. Therefore there exist finitely many indices $i=1,2, \ldots, n$ with $O_{X} U_{p_{i}} \in \alpha$ for all $i=$ $1,2, \ldots, n$ and $\operatorname{cl}_{E X}(G) \backslash G \subseteq \bigcup_{i=1}^{n}\left(E X \backslash O_{X} U_{p_{i}}\right)=E X \backslash \bigcap_{i=1}^{n} O_{X} U_{p_{i}}=$ $E X \backslash O_{X}\left(\cap_{i=1}^{n} U_{p_{i}}\right)=E X \backslash O_{X} U_{1}$, where $U_{1}=\bigcap_{i=1}^{n} U_{p_{i}}$ is open in $X$. Obviously, $O_{X}\left(U_{0} \cap U_{1}\right)=O_{X} U_{0} \cap O_{X} U_{1} \in \alpha$. Also, $O_{X} U_{1} \cap \mathrm{cl}_{E X}(G)=\left(O_{X} U_{1} \cap G\right) \cup$ $\left(O_{X} U_{1} \cap\left(\operatorname{cl}_{E X}(G) \backslash G\right)\right)=O_{X} U_{1} \cap G$. So $O_{X}\left(U_{0} \cap U_{1}\right) \subseteq G$. Take $U=U_{0} \cap U_{1}$. 
Then $U$ is open in $X$ (in fact regular open in $X$ ), and $O_{X} U \subseteq G \in \alpha$. Thus, again, $\alpha \in o_{\sigma E X}\left(O_{X} U\right) \subseteq o_{\sigma E X}(G)$, and the result follows.

(1.20) Remark. Iliadis and Fomin [8] shows that $E h X=\beta E X$ for every $H$-closed extension $h X$ of a space $X$. Porter, Vermeer and Woods [17] showed that $E_{\kappa} X=\kappa E X$ if and only if $X$ is $H$-closed. Porter and Votaw [16] proved that $\sigma E X=E \sigma X$ if and only if the set of nonisolated points of $E X$ is compact. Katětov [9] proved that every closed nowhere dense subset of a space is compact if and only if the set of nonisolated points of $X$ is compact. From [10] and [16] it follows that $\sigma E X=E \sigma X$ if and only if every closed and nowhere dense subset of $E X$ is compact. In [13] it is proved that $P \kappa X=\kappa P X$ for every space $X$. Below, in 1.21 and 1.22 , we shall briefly describe these homeomorphisms explicitly. These homeomorphisms will be used in the sequel (see 2.13, 2.15).

(1.21) Suppose that every closed and nowhere dense subset of $E X$ is compact. We construct a homeomorphism $\phi$ from $\sigma E X$ onto $E \sigma X$ as follows. Let $\alpha \in E X$. Then $\alpha^{*}=\{G \in \tau(\sigma X): G \cap X \in \alpha\}$ is a convergent open ultrafilter on $\sigma X$ such that $\operatorname{ad}_{X}(\alpha)=\operatorname{ad}_{\sigma X}\left(\alpha^{*}\right)$. If $\alpha \in \sigma E X \backslash E X$, then $\left(\alpha^{\rightarrow}\right)^{*}$ is an open ultrafilter on $\sigma X$ which converges to a point in $\sigma X \backslash X$. An easy verification (using 1.6, $1.7,1.8,1.10$, and 1.17 ) shows that the mapping $\phi: \sigma E X \rightarrow E \sigma X$ given by

$$
\phi(\alpha)= \begin{cases}\alpha^{*}, & \alpha \in E X, \\ \alpha^{\rightarrow *}, & \alpha \in \sigma E X \backslash E X,\end{cases}
$$

is well defined, one-to-one, and onto. Now let $\mathrm{iU} \in \tau(X)$, and let $V \in \tau(\sigma X)$. Then, using 1.12 and 1.14 , we obtain

(a) $\phi\left[O_{X}(V \cap X)\right]=\phi(E X) \cap O_{\sigma X} V$,

(b) $\phi\left[O_{X} U\right]=\phi(E X) \cap O_{\sigma X}\left(o_{\sigma X}(U)\right)$, and

(c) $\phi(E X)=k_{\sigma X}^{-}(X)$.

From (a), (b), and (c) it follows that $\left.\phi\right|_{E X}: E X \rightarrow k_{\sigma X}^{\leftarrow}(X)$ is a homeomorphism, and that $\phi(E X)$ is dense in $E \sigma X$. Now suppose that $G \in \tau(E X), V \in \tau(\sigma X)$, and $U \in \tau(X)$. A routine verification using 1.18 shows that

(d) $\phi\left[\sigma_{\sigma E X}(G)\right] \subseteq O_{\sigma X}\left[\sigma_{\sigma X}\left(k_{X}^{\#}(G)\right)\right]$

(e) $\phi\left[\sigma_{\sigma E X}\left(O_{X}(V \cap X)\right)\right]=O_{o X} V$, and

(f) $\phi\left[o_{\sigma E X}\left(O_{X} U\right)\right]=O_{\sigma X}\left(o_{\sigma X}(U)\right)$.

In particular, it will follow that $\phi$ is continuous. From 1.19, it follows that the family $\left\{\sigma_{\sigma E X}\left(O_{X} U\right): U \in \tau(X)\right\}$ forms an open base for $\sigma E X$. This fact, combined with (f), now shows that $\phi$ is an open mapping, and hence a homeomorphism.

(1.22) Let $X$ be a Hausdorff space. We describe explicitly a homeomorphism $\psi$ from $\kappa P X$ onto $P_{\kappa} X$. For $\alpha \in P X$, let $\alpha^{*}=\{G \in \tau(\kappa X): G \cap X \in \alpha\}$. Then 
from 1.6, 1.7, 1.8, 1.10, and 1.17 it follows that the map $\psi: \kappa P X \rightarrow P \kappa X$ given by

$$
\psi(\alpha)= \begin{cases}\alpha^{*}, & \alpha \in P X, \\ \alpha^{\rightarrow *}, & \alpha \in \kappa P X \backslash P X,\end{cases}
$$

is a well defined bijection. Further, for each $A, U \in \tau(X)$ and $V, B \in \tau(\kappa X)$ one can show (using 1.16 and 1.17 ) that

(a) $\psi\left[\pi_{X}{ }^{-}(A)\right]=\pi_{\kappa}{ }^{\leftarrow}(A)$,

(b) $\psi\left[O_{X} U\right]=\left(O_{\kappa X} U\right) \cap \psi(P X)$,

(c) $\psi(P X)=\pi_{\kappa} \stackrel{\leftarrow}{(}(X)$

(d) $\left(O_{\kappa X} V\right) \cap \phi(P X)=\psi\left(O_{X}(V \cap X)\right)$, and

(e) $\pi_{\kappa}{ }^{\leftarrow}(B) \cap \psi(P X)=\psi\left(\pi_{X}^{\leftarrow}(B \cap X)\right)$.

These facts show that $\left.\psi\right|_{P X}: P X \rightarrow \psi(P X)$ is a homeomorphism, and that $\psi(P X)$ is open and dense in $P_{\kappa} X$. The continuity of $\psi$ follows from the fact that if $\alpha \in \kappa P X$, and if $\left(O_{\kappa X} V\right) \cap \pi_{\kappa} \leftarrow(B)$ is any basic open neighborhood of $\psi(\alpha)$ in $P \kappa X$, where $V, B$ are open subsets of $\kappa X$, then $\psi\left[o_{\kappa P X}\left(O_{X}(V \cap X)\right)\right]=O_{\kappa X} V$, and, for $\alpha \in \kappa P X \backslash P X$, the set $\pi_{X}{ }^{-}(B \cap X) \cup\{\alpha\}$ is an open neighborhood of $\alpha$ in $\kappa P X$ such that $\psi\left[\left(\pi_{X}^{-}(B \cap X)\right) \cup\{\alpha\}\right] \subseteq \pi_{\kappa X}^{\leftarrow}(B)$. To show that $\psi$ is open, it suffices to show that $\psi(U \cup\{\alpha\})$ is open in $P_{\kappa} X$ for each open subset $U \subseteq P X$, and for each $\alpha \in \kappa P X \backslash P X$ such that $U \in \alpha$. If $\lambda \in \psi(U \cup\{\alpha\}) \cap$ $\pi_{\kappa} \overleftarrow{X}(X)=\psi(U)$, then $\psi(U)$ is an open neighborhood of $\lambda$ in $P \kappa X$ contained in $\psi(U \cup\{\alpha\})$. Also, since $U \in \alpha$, we have $\pi_{X}^{\#}(U) \in \alpha^{\rightarrow}$, and $\pi_{\kappa X}\left(\pi_{X}^{\#}(U)\right)=$ $\psi\left[\pi_{X}{ }^{-}\left(\pi_{X}^{\#}(U)\right)\right]=\psi(U)$. Also, $\pi_{X}^{\#}(U) \cup\left\{\alpha^{\rightarrow}\right\}$ is an open neighborhood of $\alpha^{\rightarrow}$ in $\kappa X$ and, moreover, $\pi_{\kappa X}^{\leftarrow}\left(\alpha^{\rightarrow}\right)=\alpha^{*}=\psi(\alpha)$. Thus, $\pi_{\kappa X} \overleftarrow{ }\left[\pi_{X}^{\#}(U) \cup\left\{\alpha^{\rightarrow}\right\}\right]$ is an open neighborhood of $\alpha^{\rightarrow *}$ in $P \kappa X$ contained in $\psi(U \cup\{\alpha\})$. Hence each point of $\psi(U \cup\{\alpha\})$ is an interior point in $P \kappa X$. Hence $\psi(U \cup\{\alpha\})$ is open in $P \kappa X$. Thus $\psi$ is an homeomorphism.

\section{Almost realcompact spaces}

Almost realcompact spaces were introduced and investigated by Frolik [5], and almost realcompactifications were investigated by Liu and Strecker [12]. Properties of almost realcompact spaces can be found in [5], [6], [7], [12], and [22].

(2.1) Definition. (a) An open filter $\mathscr{F}$ on a space $X$ is said to have the countable closure intersection property (abbreviated c.c.i.p.) in $X$ if for each countable subcollection $\mathscr{A} \subseteq \mathscr{F}, \bigcap\left\{\mathrm{cl}_{X}(A): A \in \mathscr{A}\right\} \neq \varnothing$.

(b) [5] A Hausdorff space $X$ is called almost realcompact if every open ultrafilter on $X$ with c.c.i.p. in $X$ converges.

(c) [12] An almost realcompactification of a space $X$ is a space $Y$ such that $Y$ is almost realcompact and $X$ is a dense subspace of $Y$. 
(d) [12] An extension $Y$ of a space $X$ is called a $\rho$-extension of $X$ provided that $Y$ is almost realcompact, and that, if $Z$ is any almost realcompactification of $X$, then there is a continuous mapping $g: Y \rightarrow Z$ such that $\left.g\right|_{X}=\iota_{X}$.

(2.2) Lemma. Let $X$ and $Y$ be spaces and let $f: X \rightarrow Y$ be a perfect, irreducible and $\theta$-continuous mapping from $X$ onto $Y$. Then:

(a) If $\mathscr{U}$ is an open ultrafilter on $X$ with c.c.i.p. in $X$, then $\mathscr{U} \rightarrow$ is an open ultrafilter on $Y$ with c.c.i.p. in $Y$, and

(b) If $\mathscr{V}$ is an open ultrafilter on $Y$ with c.c.i.p. in $Y$, then $\mathscr{V}^{-}$is an open ultrafilter on $X$ with c.c.i.p. in $X$.

Proof. The proof of (a) is easy and is left as an exercise to the reader. To prove (b), observe that by $1.7, \mathscr{V}^{-}$is an open ultrafilter on $X$. Let $\left\{V_{i}: i=1,2,3, \ldots\right\}$ be a countable subfamily of $\mathscr{V}^{-}$. Without loss of generality, assume that $V_{n} \supseteq V_{n+1}$ for all $=1,2,3, \ldots$ Then $\left\{f^{\#}\left(V_{i}\right): i=1,2, \ldots\right\}$ is a countable subfamily of $\mathscr{V}$ and hence, by hypothesis, $\cap\left\{\operatorname{cl}_{Y}\left[f^{\#}\left(V_{i}\right)\right]: i=1,2,3, \ldots\right\} \neq \varnothing$. Let $p \in \bigcap\left\{\mathrm{cl}_{Y}\left[f^{\#}\left(V_{i}\right)\right]: i=1,2,3, \ldots\right\}$. Then $f^{-}(p)$ is a nonempty compact subset of $X$. If $f^{-}(p) \cap \cap\left\{\mathrm{cl}_{X}\left(V_{i}\right): i=1,2,3, \ldots\right\}=\varnothing$, then $f^{-}(p) \subseteq \cup\{X \backslash$ $\left.\mathrm{cl}_{X}\left(V_{i}\right): i=1,2,3, \ldots\right\}$. Since the $V_{n}$ 's are decreasing, and since $f^{-}(p)$ is compact, there is a positive integer $n_{0}$ such that $f^{-}(p) \subseteq$ $X \backslash \mathrm{cl}_{X}\left(V_{n_{0}}\right)$, and $V_{n_{0}} \in \mathscr{V}^{\leftarrow}$. But then $\left.p \in \operatorname{cl}_{Y}\left(V_{i}\right): i=1,2,3, \ldots\right\} \neq \varnothing$, and, hence, $\mathscr{V} \leftarrow$ has c.c.i.p. in $X$. The proof of the proposition is now complete.

(2.3) Theorem. Let $X$ and $Y$ be spaces and $f$ a perfect, irreducible and $\theta$-continuous mapping from $X$ onto $Y$. Then $X$ is almost realcompact if and only if $Y$ is almost realcompact.

Proof. The proof follows directly from 1.6, 1.7, and 2.2 .

(2.4) Corollary. (a) A space $X$ is almost realcompact if and only if $X_{s}$ is almost realcompact.

(b) $A$ space $X$ is almost realcompact if and only if $P X$ is almost realcompact, if and only if $E X$ is almost realcompact.

(2.5) COROLlaRY. Let $Y$ be an almost realcompactification of $X$, and let $Z$ be a space with the same underlying set as $Y$ such that $\tau\left(Y^{\#}\right) \subseteq \tau(Z) \subseteq \tau\left(Y^{+}\right)$. Then (by 1.12(d)) $Z$ is almost realcompact if and only if $Y$ is almost realcompact.

In [12] Liu and Strecker constructed an almost realcompactification $\rho X$ of a space $X$. In fact, $\rho X=X \cup\{\mathscr{U} \in \mathbf{F}(X)$ : $\mathscr{U}$ has c.c.i.p. in $X\}$ as a set with subspace topology induced by $\tau(\kappa X)$. The following results are proved in [12]. 
(2.6) $\rho X$ is a projective maximum in the class of realcompactifications of $X$.

(2.7) $\rho X$ is the largest $\rho$-extension of $X$.

(2.8) $\rho x$ is the smallest almost realcompact space between $X$ and $\kappa X$.

In what follows, we discuss the interplay between the absolutes and almost realcompactifications. We first state the following corollary.

(2.9) Corollary. E $\rho X$ and $P \rho X$ are almost realcompactifications of $E X$ and $P X$ respectively. Moreover, $E \rho X$ is realcompact (see [22]).

(2.10) The space $\beta E X$ can also be realized as the space $\Theta X=\{\mathscr{U}: \mathscr{U}$ is an open ultrafilter on $X\}$ whose topology has the open base $\left\{O_{X} U: U \in \tau(X)\right\}$, where $O_{X} U=\{\mathscr{U} \in \Theta X: U \in \mathscr{U}\}$ (see [19].) Now, let $\rho X$ be the almost realcompactification of $X$ (see [12].) Let $\mathscr{U} \in \Theta X$, and let $\mathscr{U}^{*}=\{G \in \tau(\kappa X)$ : $G \cap X \in \mathscr{U}\}$. Then $\mathscr{U}^{*}$ is an open ultrafilter on $\kappa X$ and $\operatorname{ad}_{\kappa X}\left(\mathscr{U}^{*}\right)=\operatorname{ad}_{\kappa}(\mathscr{U})$ consists of exactly one point. Define $\psi_{\kappa}: \quad \Theta X \rightarrow \kappa X$ by $\psi_{\kappa X}(\mathscr{U})=\operatorname{ad}_{\kappa X}\left(\mathscr{U}^{*}\right)$. If $\mathscr{U} \in \Theta X \backslash E X$, then $\mathscr{U} \in \kappa X \backslash X$ and $\mathcal{O}_{\kappa X}^{\mathscr{U}}=\mathscr{U}$. Since $\psi_{\kappa X}(\mathscr{U})=p$ if and only if $\mathcal{O}_{\kappa X}^{P} \subseteq \mathscr{U}$ (for each $\mathscr{U} \in \Theta X \backslash E X$ ), it follows that the map $\psi_{\kappa}$ can be equivalently defined as

$$
\psi_{\kappa X}(\mathscr{U})= \begin{cases}k_{X}(\mathscr{U}), & \mathscr{U} \in E X, \\ \mathscr{U}, & \mathscr{U} \in \Theta x \backslash E X,\end{cases}
$$

where $k_{X}$ is the usual map from $E X$ onto $X$. Then $\psi_{\kappa}$ is a perfect, irreducible and $\theta$-continuous mapping from $\Theta X$ onto $\kappa X$. Now, let

$$
E_{\rho} X=\{\mathscr{U} \in \Theta X: \mathscr{U} \text { has c.c.i.p. relative to } X\} \text {. }
$$

Obviously, $E X \subseteq E_{\rho} X, E_{\rho} X$ is a dense of $\Theta X$, and $E X$ is dense in $E_{\rho} X$.

(2.11) Proposition. For every space $X, E_{\rho} X$ is a realcompactification of $E X$, and $E_{\rho} X=E \rho X$.

Proof.

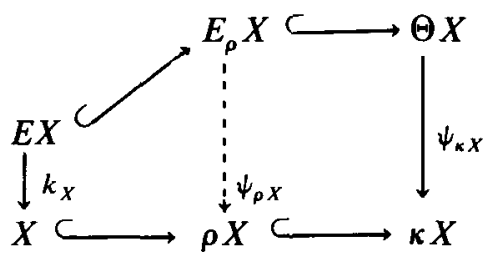

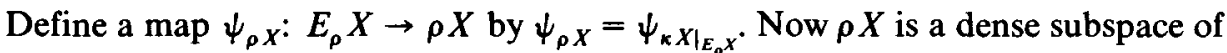
$\kappa X, E_{\rho} X$ is a dense subspace of $\Theta X$, and $E_{\rho} X=\psi_{\kappa X}^{\leftarrow}(\rho X)$. Hence, by 1.1 and $1.2, \psi_{\rho} X$ is a perfect, irreducible, and $\theta$-continuous mapping from $E_{\rho} X$ onto $\rho X$. Hence, by $1.15, E_{\rho} X=E \rho X$. Since $\rho X$ is almost realcompact, $E_{\rho} X$ is realcompaet by 2.4 . 
(2.12) Note. By 2.6, $\rho E X$ is a projective maximum in the class of all the almost realcompactifications of $E X$. Hence there exists a continuous mapping $g: \rho E X \rightarrow$ $E_{\rho} X(=E \rho X)$ that fixes the points of $E X$. We note that $E \rho X$ carries the subspace topology from $\beta E X$, whereas $\rho E X$ carries the subspace topology from $\kappa E X$. Also, $E X \hookrightarrow v E X \hookrightarrow E \rho X=E_{\rho} X \hookrightarrow \beta E X$, where each embedding is dense and where, for a Tychonoff space $Z, \beta Z$ and $v Z$ have their usual meaning. We now regard $\rho X$ as the subspace of $\sigma X$ given by the points of $\rho X$ and denote the space ( $\rho X$, $\left.\left.\tau(\sigma X)\right|_{\rho X}\right)$ by $\zeta X$.

(2.13) TheOREM. Let $X$ be a Hausdorff space such that every closed and nowhere dense subset of $E X$ is compact. Then $E \zeta X=\zeta E X$.

Proof. We consider the homeomorphism $\phi$ from $\sigma E X \rightarrow E \sigma X$ given by 1.21 . Let $\delta \in \zeta E X \backslash E X$. Then $\delta \in \sigma E X \backslash E X$. Hence $\phi(\delta)=\delta^{* *} \in E \sigma X \backslash \phi(E X)$. Suppose $\delta \rightarrow *$ does not converge to a point in $\zeta X$. Then $\alpha=\operatorname{ad}_{\sigma X}\left(\delta^{*}\right)=$ $k_{\sigma X}\left(\delta^{*}\right) \in \sigma X \backslash \zeta X$. Let $G \in \alpha$. Then $\alpha \in o_{\sigma X}(G)$. So, $o_{\sigma X}(G) \cap U \neq \varnothing$ for all $U \in \delta^{* *}$ and, since $\delta^{* *}$ is an open ultrafilter on $\sigma X$, it follows that $o_{o X}(G) \in \delta \rightarrow *$. Hence, $G \in\left(\delta^{* *}\right)_{*}=\delta \rightarrow$. Thus, $\alpha \subseteq \delta \rightarrow$. Since both $\alpha$ and $\delta \rightarrow$ are open ultrafilters on $X$, it follows that $\alpha=\delta \rightarrow$. Now $\delta$ has the c.c.i.p. in $E X$. So, by (2.2), $\alpha$ has c.c.i.p. in $X$, whence $\alpha \in \zeta X$, a contradiction. Thus $\delta \rightarrow *$ converges to a point in $\zeta X$. Consequently, $\delta=\left\{G \cap \zeta X: G \in \delta^{*}\right\} \in E \zeta X$. Let $\phi_{0}=\left.\phi\right|_{\zeta E X}$ be given by

$$
\phi_{0}(\delta)= \begin{cases}\delta, & \delta \in E X, \\ \delta, & \delta \in \zeta E X \backslash E X .\end{cases}
$$

Then $\phi_{0}$ is a one-to-one mapping from $\zeta E X$ into $E \zeta X$. To show that $\phi_{0}$ is onto, let $\lambda \in E \zeta X \backslash E X$. Then $\lambda^{0}=\{G \in \tau(\sigma X): G \cap \zeta X \in \lambda\}$ is an open ultrafilter on $\sigma X$. Hence $\lambda^{0}=\alpha^{*}$ for some $\alpha \in \sigma E X$, and $\lambda_{*}^{0}=\left(\alpha^{*}\right)_{*}=\alpha^{\rightarrow}$ is a free open ultrafilter on $X$, since $\alpha \rightarrow \zeta X$. So $\alpha \rightarrow$ has c.c.i.p. in $X$. Hence $\alpha=(\alpha \rightarrow)^{\leftarrow}$ has c.c.i.p. in $E X$. Therefore $\alpha \in \zeta E X \backslash E X$ and $\lambda=\hat{\alpha}$. Thus $\phi_{0}$ is onto. Since $\zeta E X$ is dense in $\sigma E X, E \zeta X$ is dense in $E \sigma X, \phi_{0}$ is a bijection, and $\phi$ is a homeomorphism, and so the theorem follows.

(2.14). TheOREM. For every space $X, \zeta P X=P \zeta X$.

ProOF.

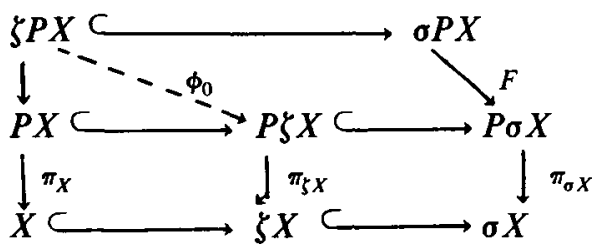


We consider the homeomorphism $F$ from $\sigma P X$ onto $P a X$ given by

$$
F(\delta)= \begin{cases}\delta, & \delta \in P X, \\ \delta^{\#}, & \delta \in \sigma P X \backslash P X,\end{cases}
$$

where $\delta^{\#}=\left\{U \subseteq \sigma X\right.$ : is open, $\left.O_{\sigma X} U \cap P X \in \delta\right\}$ (see [17, Theorem 4.2]). Let $\delta \in \zeta P X \backslash P X$. Then $\delta \in \sigma P X$, and $F(\delta)=\delta^{\#} \in P \sigma X \backslash P X$. Let $\alpha=\operatorname{ad}_{\sigma}\left(\delta^{\#}\right)$. Then $\alpha=\left\{G \in \tau(X)\right.$ : there is a $U \in \delta^{\#}$ such that $\left.G=U \cap X\right\}$ is an open ultrafilter on $X$. We will show that $\alpha \in \zeta X$. Suppose $\alpha \notin \zeta X$. Then there exists a countable subfamily $\left\{G_{i}: i \in \omega\right\} \subseteq \alpha$ such that

$$
\varnothing=\bigcap_{i \in \omega} \mathrm{cl}_{X}\left(G_{i}\right)=\bigcap_{i \in \omega}\left[X \cap \mathrm{cl}_{\sigma X}\left(G_{i}\right)\right] .
$$

On the other hand, $P X \cap O_{\sigma}\left(o_{o}\left(G_{i}\right)\right) \in \delta$ for all $i \in \omega$, and $\delta$ has c.c.i.p. in $P X$. Hence,

$$
\begin{aligned}
\varnothing & \neq \pi_{\sigma X}\left[P X \cap \bigcap_{i \in \omega} o_{\sigma X}\left(\sigma_{\sigma X}\left(G_{i}\right)\right)\right] \subseteq \pi_{\sigma X}(P X) \cap \bigcap_{i \in \omega} \pi_{\sigma X}\left(O_{\sigma X}\left(o_{\sigma X}\left(G_{i}\right)\right)\right) \\
& =\bigcap_{i \in \omega}\left[X \cap \mathrm{cl}_{\sigma X}\left(o_{\sigma X}\left(G_{i}\right)\right)\right]=\bigcap_{i \in \omega} \mathrm{cl}_{X}\left(G_{i}\right),
\end{aligned}
$$

a contradiction. Thus $\alpha \in \zeta X$. In particular, it follows that $\hat{\delta}=\left.\delta^{\#}\right|_{\zeta X}$ is a convergent open ultrafilter on $\zeta Z$ and hence $\hat{\delta} \in P \zeta X$. So, define $F_{0}$ by

$$
F_{0}(\delta)= \begin{cases}\delta, & \delta \in P X \\ \delta, & \delta \in \zeta P X \backslash P X .\end{cases}
$$

Then $F_{0}$ is a one-to-one mapping from $\zeta P X$ to $P \zeta X$. We now show that $F_{0}$ is onto. Let $\lambda \in P \zeta X \backslash P X$. Then $\alpha=\pi_{\zeta X}(\lambda) \in \zeta X \backslash X, \alpha=\{U \cap X: U \in \lambda\}$, and $\alpha^{*}=\lambda^{*}=\delta^{\#}$ (where * is taken with respect to $\sigma X$ ) for some $\delta \in \sigma P X$. To show that $\delta \in \zeta P X$, we show that $\delta=\alpha^{-}$. It suffices to show that $\delta^{\#}=\left(\alpha^{-}\right)^{\#}$, or, alternatively, that $\left(\delta^{\#}\right)_{*}=\left(\left(\alpha^{-}\right)^{\#}\right)_{*}=\alpha$. Obviously, $\left(\delta^{\#}\right)_{*}=\left(\alpha^{*}\right)_{*}=\alpha$. Also, $\left(\left(\alpha^{\leftarrow}\right)^{\#}\right)_{*}=\alpha$. Obviously, $\left(\delta^{\#}\right)_{*}=\left(\alpha^{*}\right)_{*}=\alpha$. Also, $\left(\left(\alpha^{\leftarrow}\right)^{\#}\right)_{*}=\{W \subseteq X$ : $W$ is open, $W=U \cap X$ for some open $U \subseteq \sigma X$ and $\left.O_{\sigma X} U \cap P X \in \alpha^{\leftarrow}\right\}$. Since for every $U \in \tau(\sigma X), O_{\sigma X} U=O_{\sigma X}\left[o_{\sigma X}\left(\right.\right.$ int $\left._{X} \mathrm{cl}_{X}(U \cap X)\right)$ ], it follows that $P X \cap$ $O_{\sigma X} U=O_{X}\left(\right.$ int $\left._{X} \mathrm{cl}_{X} W\right)=O_{X} W$ and hence, by 1.17 , that $\left(\left(\alpha^{\leftarrow}\right)^{\#}\right)_{*}=\left(\alpha^{\leftarrow}\right)^{\rightarrow}=$ $\alpha$. Since $\hat{\delta}=\lambda$, it follows that $F_{0}$ is onto. Now $\zeta P X$ is dense in $\sigma P X, P \zeta X$ is dense in $\operatorname{Po} X, F_{0}=\left.F\right|_{\zeta P X}$, and $F_{0}$ is a bijection, so that $F_{0}$ is a homeomorphism, and the theorem follows.

\section{(2.15) THEOREM. For every space $X, \rho P X=P \rho X$.}

Proof. We consider the homeomorphism $\psi$ from $\kappa P X$ onto $P \kappa X$ described in 1.22. If $\delta \in \rho P X \backslash P X$, then $\delta \in \kappa P X$, and $\psi(\delta)=\delta^{* *} \in P \kappa X \backslash P X$. If $\alpha=$ $\pi_{\kappa X}\left(\delta^{*}\right)$, then $\alpha \in \kappa X \backslash X$. Moreover, $\alpha=\delta^{\rightarrow}$, and hence, by 2.2, $\alpha$ has c.c.i.p. in $X$; and so $\alpha \in \rho X \backslash X$. Let $\hat{\delta}=\{G \subseteq \rho X: G \cap X \in \alpha\}$. Then $\hat{\delta}$ is an open 
ultrafilter on $\rho X$ with c.c.i.p. in $\rho X$. Since $\rho X$ is almost realcompact, $\hat{\delta}$ converges in $\rho X$, and hence $\delta \in P \rho X$. Define a map $\psi_{0}: \rho P X \rightarrow P \rho X$ by

$$
\psi_{0}(\delta)= \begin{cases}\delta, & \delta \in P X, \\ \delta, & \delta \in \rho P X \backslash P X .\end{cases}
$$

Reasoning similar to that used in (2.13) shows that $\psi_{0}$ is a one-to-one mapping from $\rho P X$ onto $P \rho X$. The result now follows from the fact that $\rho P X$ is dense in $\kappa P X, P \rho X$ is dense in $P \kappa X, \psi$ is a homeomorphism, and $\psi_{0}=\left.\psi\right|_{\rho P X}$.

\section{Acknowledgement}

This is a part of the doctoral work done at the University of Kansas under the supervision of Proferror Jack Porter. The author is extremely grateful to Professor Porter for his invaluable help and suggestions. The author also thanks the referee for several useful suggestions.

\section{References}

[1] B. Banaschewski, 'Extensions of topological spaces', Canad. Math. Bull. 7 (1964), 1-22.

[2] B. Banaschewski, 'Projective covers in categories of topological spaces and topological algebras', Proceedings of the Kanpur Topological Conference, pp. 63-91 (Czechoslovak Academy of Sciences, Academia, Prague 1971).

[3] R. F. Dickman Jr., J. R. Porter and L. R. Rubin, 'Completely regular absolutes and projective objects', Pacific J. Math. 94 (1981), 277-295.

[4] S. Fomin, 'Extensions of topological spaces', Ann. of Math. 44 (1943), 471-480.

[5] Z. Frolík, 'On almost realcompact spaces', Bull. Acad. Polon. Sci. Sér. Sci. Math. Phys. Astronom. 49 (1961), 247-250.

[6] Z. Frolik, 'A generalization of realcompact spaces', Czech. Math. J. 13 (1963), 127-138.

[7] Z. Frolik and C. T. Liu, 'An embedding characterization of almost realcompact spaces', Proc. Amer. Math. Soc. 32 (1972), 294-298.

[8] S. Iliadis and S. Fomin, 'The method of centered systems in the theory of topological spaces', Uspehi Mat. Nauk 21 (1966), 47-76. (English translation: Russian Math. Surveys 21 (1966), 37-62.)

[9] M. Katětov, 'Über $H$-abgeschlossene und bikompacte Räume', Časopis Pěst. Math. Fys. 69 (1940), 36-49.

[10] M. Katětov, 'On the equivalence of certain types of extensions of topological spaces', Časopis Pěst. Mat. Fys. 72 (1947), 101-106.

[11] C. T. Liu, 'The $\alpha$-closure $\alpha X$ of a topological space $X$ ', Proc. Amer. Math. Soc. 23 (1969), 605-607.

[12] C. T. Liu and G. E. Strecker, 'Concerning almost realcompactifications', Czech. Math. J. 22 (1977), 181-190.

[13] J. Mioduschewski and L. Rudolf, ' $H$-closed and extemally disconnected Hausdorff spaces', Dissertationes Math. 66 (1969), 1-55. 
[14] V. I. Ponomarev, 'On spaces co-absolute with metric spaces', Russian Math. Surveys 21 (1966), 87-114.

[15] J. Porter and C. Votaw, 'H-closed extensions I', General Topology and Appl. 3 (1973), 211-224.

[16] J. Porter and C. Votaw, ' $H$-closed extensions II', Trans. Amer. Math. Soc. 202 (1975), 193-209.

[17] J. Porter, J. Vermeer and R. G. Woods, ' $H$-closed extensions of absolutes', to appear.

[18] Jack R. Porter and R. Grant Woods, 'Minimal extremally disconnected Hausdorff spaces', General Topology and Appl. 8 (1978), 9-26.

[19] Jack R. Porter and R. Grant Woods, 'Extensions of Hausdorff spaces', Pacific J. Math. 103 (1982), 111-134.

[20] D. P. Strauss, 'Extremally disconnected spaces', Proc. Amer. Math. Soc. 18 (1976), 305-309.

[21] S. Willard, General Topology (Addison Wesley, Reading and London, 1968).

[22] R. Grant Woods, 'A Tychonoff almost real compactification', Proc. Amer. Math. Soc. 43 (1974), 200-208.

[23] R. Grant Woods, 'A survey of absolutes of topological spaces', Topological Structures II, pp. 323-362 (Math. Centre Tracts 116, Amsterdam, 1979).

\section{Department of Mathematics}

Southeast Missouri State University

Cape Girardeau, Missouri 63701

U.S.A. 